\title{
Rayleigh-Bénard convection of Casson fluids
}

\author{
M.S. Aghighi ${ }^{\mathrm{a}, *}$, A. Ammar ${ }^{\mathrm{b}}$, C. Metivier ${ }^{\mathrm{c}}$, M. Gharagozlu ${ }^{\mathrm{a}}$ \\ a Department of Mechanical Engineering, Bu-Ali Sina University, 65175-38695 Hamedan, Iran \\ ${ }^{\mathrm{b}}$ Arts et Métiers ParisTech, 2 Boulevard du Ronceray, BP 93525, F-49035 Angers Cedex 01, France \\ ${ }^{\mathrm{c}}$ LEMTA, UMR 7563, 2 Avenue de la Forêt de Haye, TSA 60604, 54518 Vandoeuvre les Nancy, France
}

Keywords:

Rayleigh-Bénard

Natural convection

Yield stress

Casson model

Finite element

\section{A B S T R A C T}

\section{Introduction}

Viscoplastic fluids, characterized by a yield stress $\tau_{y}$, are known to present a complex transition between a solid-like and a fluid-like behavior. If the material is not stressed enough, i.e. less than the yield stress, the material does not flow and behaves as a solid. Above the yield stress, it flows with a shear-thinning behavior. The most used models describing viscoplastic fluids are the inelastic Bingham [1], Herschel-Bulkley [2] and Casson [3] models. The Bingham model is the simplest model which deals with a yield stress. Due to its relative simplicity, the Bingham model is the most frequently used model in theoretical and numerical studies. However, the Bingham model is not realistic. Most of real materials behave as shear-thinning yield stress fluids such as the HerschelBulkley or Casson fluids. The Casson model is currently used in the field of food stuff [4], in particular, it has been chosen by the International Office of Cocoa and Chocolate to model the chocolate rheological behavior. Furthermore, the Casson model is often adopted in medicine to fit the blood rheology [5-10]. The flow of some particulate suspensions can also be modeled by Casson fluids [11], [12].

The Rayleigh-Bénard Convection (RBC) is a buoyancy-driven instability, i.e. it occurs in a fluid layer heated from below and submitted to a vertical temperature gradient. This configuration is widely encountered in nature and also in industrial processes, justifying the impressive volume of work devoted to its understanding since more than a century especially in the case of Newtonian fluids, reviews are provided by Refs. [13-15] and some recent studies are done by Refs. [16-20]. Compared to the Newtonian case, the RBC in viscoplastic fluids has been little studied. However, the RBC in viscoplastic fluids is experiencing a rising interest these last decades leading to theoretical and numerical studies [21-28], as well as experimental investigations [29], [30]. By considering either Bingham or Herschel-Bulkley fluids, all these studies show that the yield stress has a stabilizing effect, meaning that the conductive solution (motionless solution) remains stable for larger Rayleigh values when $\tau_{y}$ is increased. It is also shown (i) that the motionless solution is linearly stable in the case of an inelastic viscoplastic fluid [21] [22], (ii) that convection can occur if the amplitude perturbation is large enough and (iii) that the transition from conductive to convective regime is subcritical [27], [31]. Another result of great interest is the structure of the convective flow, via the velocity and temperature profiles, and the topology of the flow, i.e. the existence, size and location of the yielded and unyielded regions because it can have consequences on heat transfer for instance. Indeed, in the case of Bingham and Herschel-Bulkley fluids, the increase in $\tau_{y}$ involves a decrease in the Nusselt number as well as the development and the increase in unyielded regions [23] [26], [27]. Concerning the experimental investigations, the first to study the RBC in yield stress fluids is Darbouli et al. [29], who focus on the onset of convection in Carbopol gels in a cylindrical cavity. Onset of the Rayleigh-Bénard convection in Carbopol gels has also been studied experimentally in a rectangular cavity by Kebiche et al. [30]. In these studies, it is shown that the governing parameter is the yield number $Y$ which represents the ratio between the yield stress and the buoyant effects. However, the studies do not obtain similar critical conditions in terms of $Y_{c}$ at the onset of convection, this is certainly due to different boundary conditions. A

\footnotetext{
* Corresponding author

E-mail addresses: ms.aghighi@gmail.com (M.S. Aghighi), Amine.Ammar@ensam.eu (A. Ammar), christel.metivier@univ-lorraine.fr (C. Metivier).
} 
more recent study given by Métivier et al. [32] highlight similar critical conditions than that given by Ref. [29]. Furthermore, by considering the development of thermal plumes due to a localized heat source in Carbopol gels, Davaille et al. [33] obtain the same order of $Y_{c}$ than [29] and [32].

The aim of our paper is to consider numerically the RBC in Casson fluids since, to our best knowledge, it does not exist any result while the Casson model is widely used to describe viscoplastic fluids. For this respect, we extend the numerical study given by Turan et al. [23] and Aghighi \& Amar [26] to the Casson fluid case and we consider the bench case of a two-dimensional square cavity heated from below.

First, this paper set the mathematical formulation of our configuration. The numerical method and its validation are then described. In section 4, results are presented and discussed. Correlations for the Nusselt number are also proposed in this section. Our paper finally ends with a concluded section.

\section{Mathematical formulation}

\subsection{Casson model}

The stress-deformation behavior of viscoplastic materials based on Casson model is given by:

$\tau_{i j}=\left(1+\left(\frac{B n}{\dot{\gamma}}\right)^{\frac{1}{2}}\right)^{2} \dot{\gamma}_{i j}$ for $\tau>\tau_{y}$

and

$\dot{\gamma}=0$ for $\tau<\tau_{y}$

For $i, j=1,2$ with $\left(x_{1}, x_{2}\right)=(x, y)$ are defined in Fig. 2, Bn is Bingham number defined by

$B n=(P r / R a)^{-1 / 2} \frac{\tau_{y}}{\rho \beta g \Delta T H}$

The Prandtl number, $P r$, and Rayleigh number, $R a$, are defined as follows:

$\operatorname{Pr}=\frac{\mu C_{p}}{k}$

$R a=\frac{g \beta \Delta T H^{3}}{\alpha \nu}$

The various dimensional quantities above are defined as follows: $\tau_{y}$ is the yield stress, $\mu$ is the constant plastic viscosity, $C_{p}$ is specific heat capacity, $k$ is the thermal conductivity, $g$ is the acceleration due to gravity, $\beta$ is the coefficient of thermal expansion, $\alpha$ is the thermal

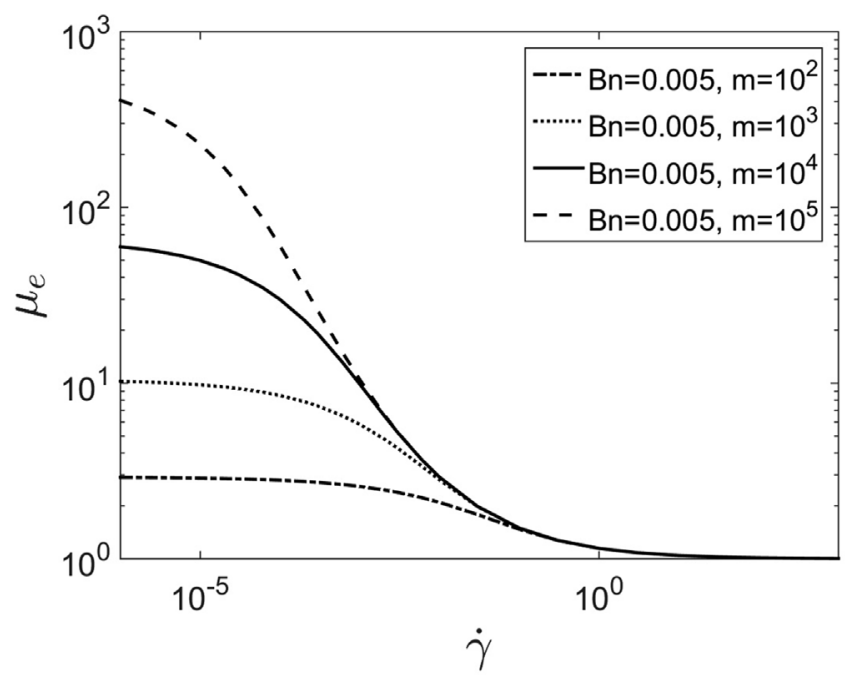

diffusivity, $\Delta T$ is the temperature difference between hot and cool walls and $\nu$ is the kinematic viscosity.

$\dot{\gamma}$ and $\tau$ are the second invariant of the respective rate-of-strain and shear stress tensors $\dot{\gamma}=\sqrt{\frac{1}{2} \dot{\gamma}_{i j} \dot{\gamma}_{i j}}$ and $\tau=\sqrt{\frac{1}{2} \tau_{i j} \tau_{i j}}$, Where $\dot{\gamma}_{i j}$ is defined by:

$\dot{\gamma}_{i j}=\frac{\partial u_{i}}{\partial x_{j}}+\frac{\partial u_{j}}{\partial x_{i}}$

Papanastasiou modifications method [34] can be applied to Casson model to avoid discontinuity between yielded and unyielded regions. This method consists in an exponential regularization of the viscosity, leading to the expression of the stress [35]:

$\tau_{i j}=\left(1+\left(\frac{B n}{\dot{\gamma}}\right)^{\frac{1}{2}}(1-\exp (-\sqrt{m \dot{\gamma}}))^{2} \dot{\gamma}_{i j}\right.$

where $m$ is the regularization parameter which is a large value. It is worth noting that, the effective viscosity, $\mu_{e}=\frac{\tau_{i j}}{\hat{\gamma}_{i j}}$, tends to $m B n$ when $\dot{\gamma}$ tends to zero (Fig. 1). This is a large but finite value which depends on the Papanastasiou parameter and the Bingham number. On the other hand, viscosity $\mu_{e}$ tends to 1 when $\dot{\gamma}$ tends to infinity. Furthermore, one notices that at fixed value of $m$, the decrease in $B n$ involves a decrease in the effective viscosity (whatever the value of $\dot{\gamma}$ ) but also a decrease in the shear-thinning degree which could be defined by $d \mu_{e} / d \dot{\gamma}$.

\subsection{Rayleigh-Bénard convection}

The configuration under analysis is depicted in Fig. 2. The model is defined in the unit square domain fulfilled by a yield stress fluid modeled by the Casson law. Because one knows that the transient phenomena do not modify the steady state result (see for instance [23] [24] [36]), in this study we consider the steady state set of equations corresponding to the two-dimensional natural convection case. Assuming the Boussinesq approximation and using the characteristic scales $H$ for length, $u_{0}=(g \beta H \Delta T)^{1 / 2}$ for the velocity, and $p_{0}=\rho u_{0}^{2}$ for the pressure, the steady dimensionless continuity, momentum and energy equations are:

$\frac{\partial u}{\partial x}+\frac{\partial v}{\partial y}=0$

$u \frac{\partial u}{\partial x}+v \frac{\partial u}{\partial y}=-\frac{\partial p}{\partial x}+\operatorname{Pr}^{\frac{1}{2}} R a^{\frac{-1}{2}}\left(\frac{\partial \tau_{x x}}{\partial x}+\frac{\partial \tau_{y x}}{\partial y}\right)$

$u \frac{\partial v}{\partial x}+v \frac{\partial v}{\partial y}=-\frac{\partial p}{\partial y}+\operatorname{Pr}^{\frac{1}{2}} R a^{\frac{-1}{2}}\left(\frac{\partial \tau_{y y}}{\partial y}+\frac{\partial \tau_{x y}}{\partial x}\right)+\theta$

$u \frac{\partial \theta}{\partial x}+v \frac{\partial \theta}{\partial y}=(R a \cdot \operatorname{Pr})^{-1 / 2}\left(\frac{\partial^{2} \theta}{\partial x^{2}}+\frac{\partial^{2} \theta}{\partial y^{2}}\right)$

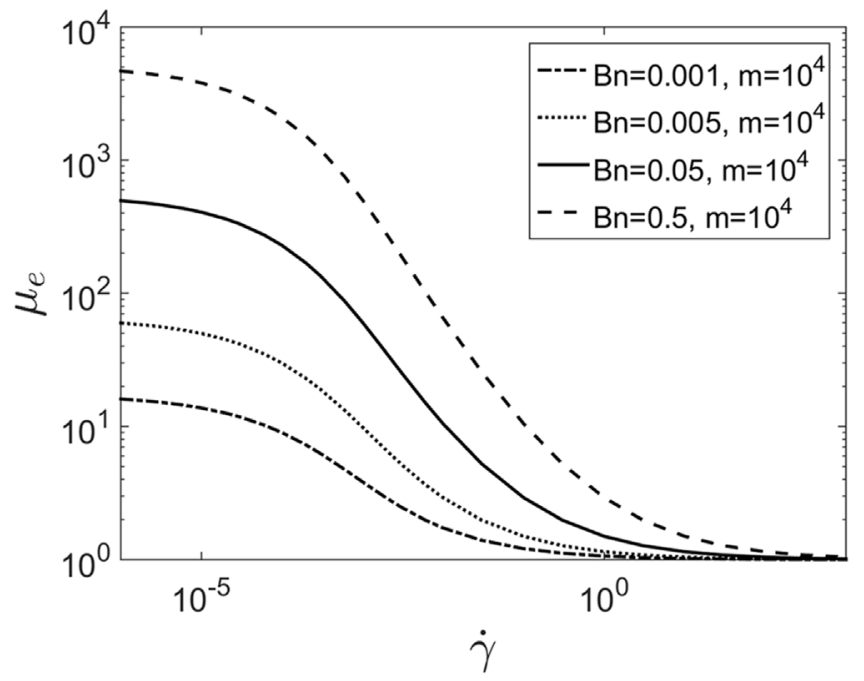

Fig. 1. Variations of viscosity $\mu$ with $\dot{\gamma}$ at the constant Bingham number (left) and constant regularization parameter (right). 


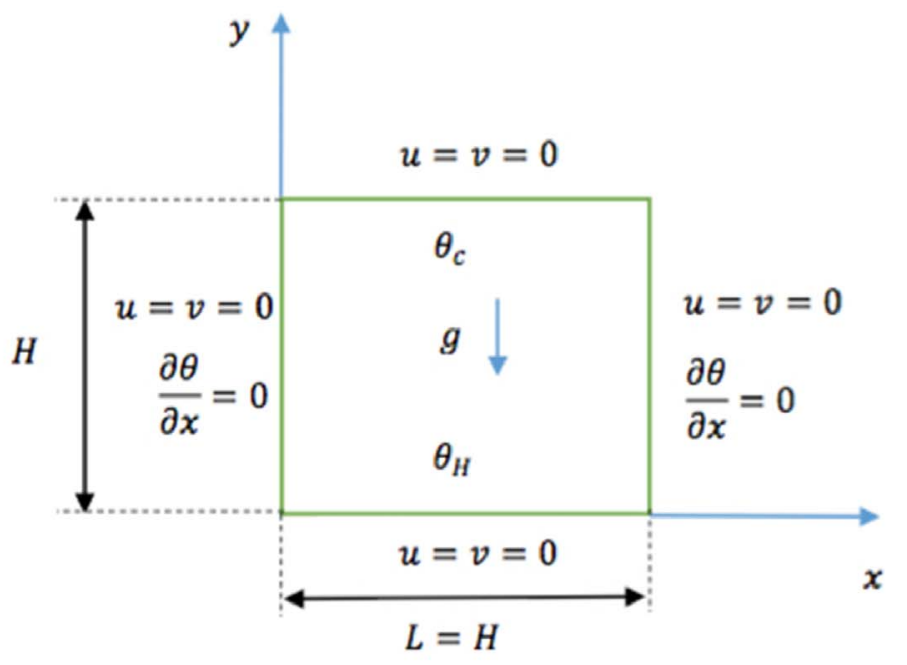

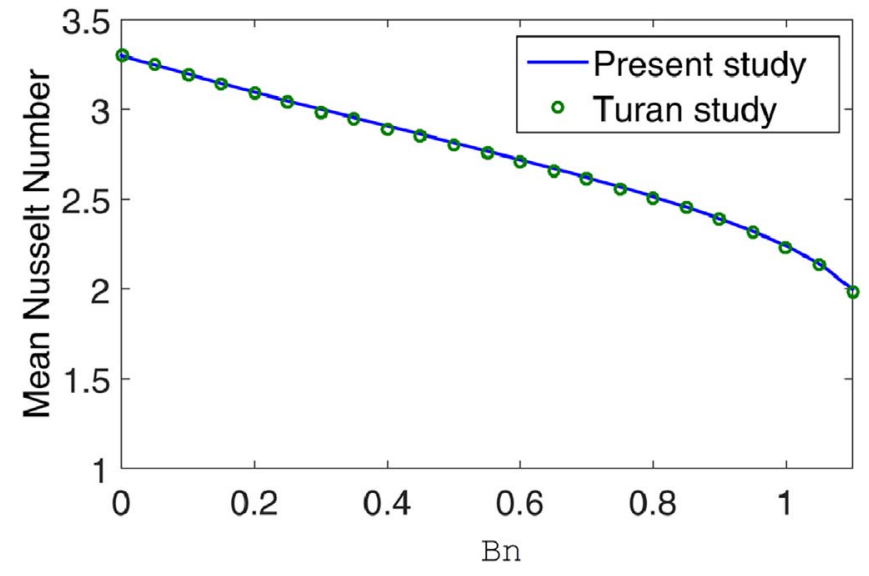

Fig. 3. Mean Nusselt number $\mathrm{Nu}$ for Bingham fluids at $5 * 10^{4}$ and $\mathrm{Pr}=10$ (line: present study, points: Turan study [23]).

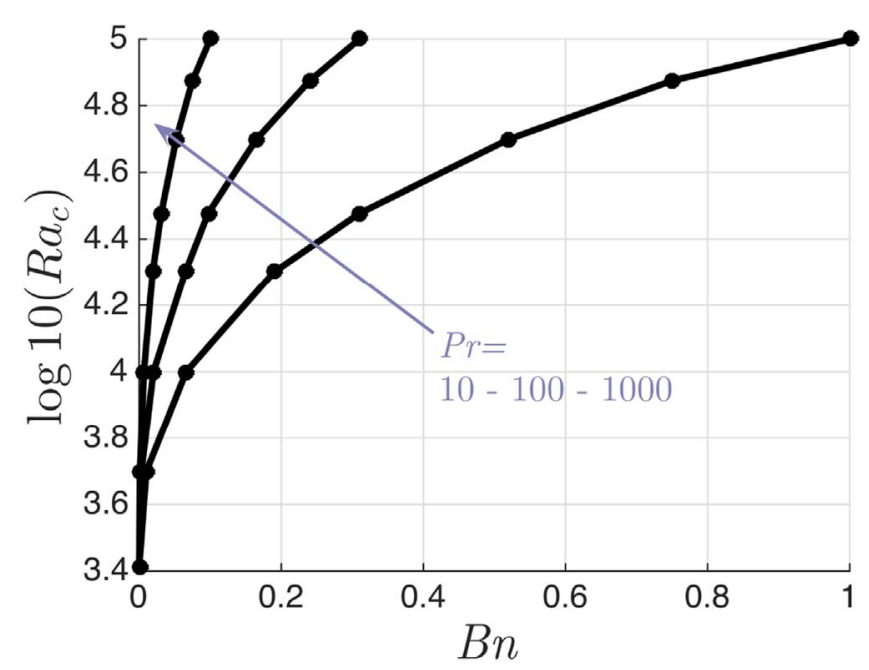

Fig. 4. The variations of critical Rayleigh number $R a_{c}$ with Bingham number $B n$ for $\operatorname{Pr}=10,100,1000$.

Where $u, v, \theta$ and $p$ are dimensionless horizontal velocity, vertical velocity, temperature and pressure respectively.

No-slip conditions are considered at walls.

$u=v=0$,
Adiabatic conditions are written at vertical walls and imposed temperatures at horizontal walls:

$\frac{\partial \theta}{\partial x}=0$ at $x=0$ and $x=1$

$\theta=0.5$ at $y=0$

$\theta=-0.5$ at $y=1$

In these equations, the dimensionless temperature $\theta$ is defined by:

$\theta=\frac{T-T_{r}}{T_{H}-T_{C}}$

Where $T_{C}$ and $T_{H}\left(T_{H}>T_{C}\right.$ ) are the temperatures enforced at the top and bottom cavity boundaries, respectively, and $T_{r}$ is a reference temperature: $T_{r}=\left(T_{H}+T_{C}\right) / 2$.

The heat flux averaged over the hot wall is defined via the Nusselt number:

$\overline{N u}=-\left.\int_{0}^{1} \frac{\partial \theta}{\partial y}\right|_{y=0} d x$

\section{Numerical method}

In order to solve the system of equation (6) with their corresponding boundary conditions (7), by using Matlab we have developed a numerical code that is presented in this section. Similarly to Aghighi et al. [37] in what follows we consider a penalty formulation of the incompressibility constraint. In this way, equation (6) can be written as follows:

$\frac{\partial u}{\partial x}+\frac{\partial v}{\partial y}-\frac{1}{\lambda} p=0$

$u \frac{\partial u}{\partial x}+v \frac{\partial u}{\partial y}+\frac{\partial p}{\partial x}=\operatorname{Pr}^{\frac{1}{2}} R a^{\frac{-1}{2}}\left(\frac{\partial \tau_{x x}}{\partial x}+\frac{\partial \tau_{y x}}{\partial y}\right)$

$u \frac{\partial v}{\partial x}+v \frac{\partial v}{\partial y}+\frac{\partial p}{\partial y}-\theta=\operatorname{Pr}^{\frac{1}{2}} R a^{\frac{-1}{2}}\left(\frac{\partial \tau_{y y}}{\partial y}+\frac{\partial \tau_{x y}}{\partial x}\right)$

$u \frac{\partial \theta}{\partial x}+v \frac{\partial \theta}{\partial y}-(R a . P r)^{-1 / 2}\left(\frac{\partial^{2} \theta}{\partial x^{2}}+\frac{\partial^{2} \theta}{\partial y^{2}}\right)=0$

Where $\lambda$ is a sufficiently large constant which allows to ensure the incompressibility condition.

The matrix form of the system of equation (10) writes:

$[L]\{T\}+\{f\}=0$ 


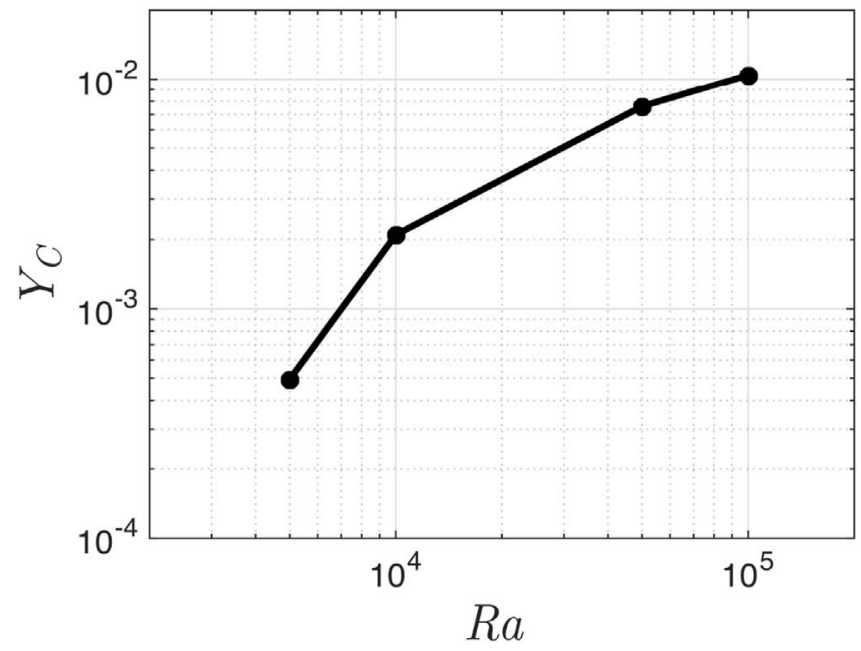

Fig. 5. The variations of critical Yield number $Y_{c}$ with Rayleigh number $R a$ for $P r=100$.

$\{T\}=\left\{\begin{array}{c}u \\ v \\ p \\ \theta\end{array}\right\} ; \quad\{f\}=\left\{\begin{array}{c}f_{x} \\ f_{y} \\ 0 \\ 0\end{array}\right\}$
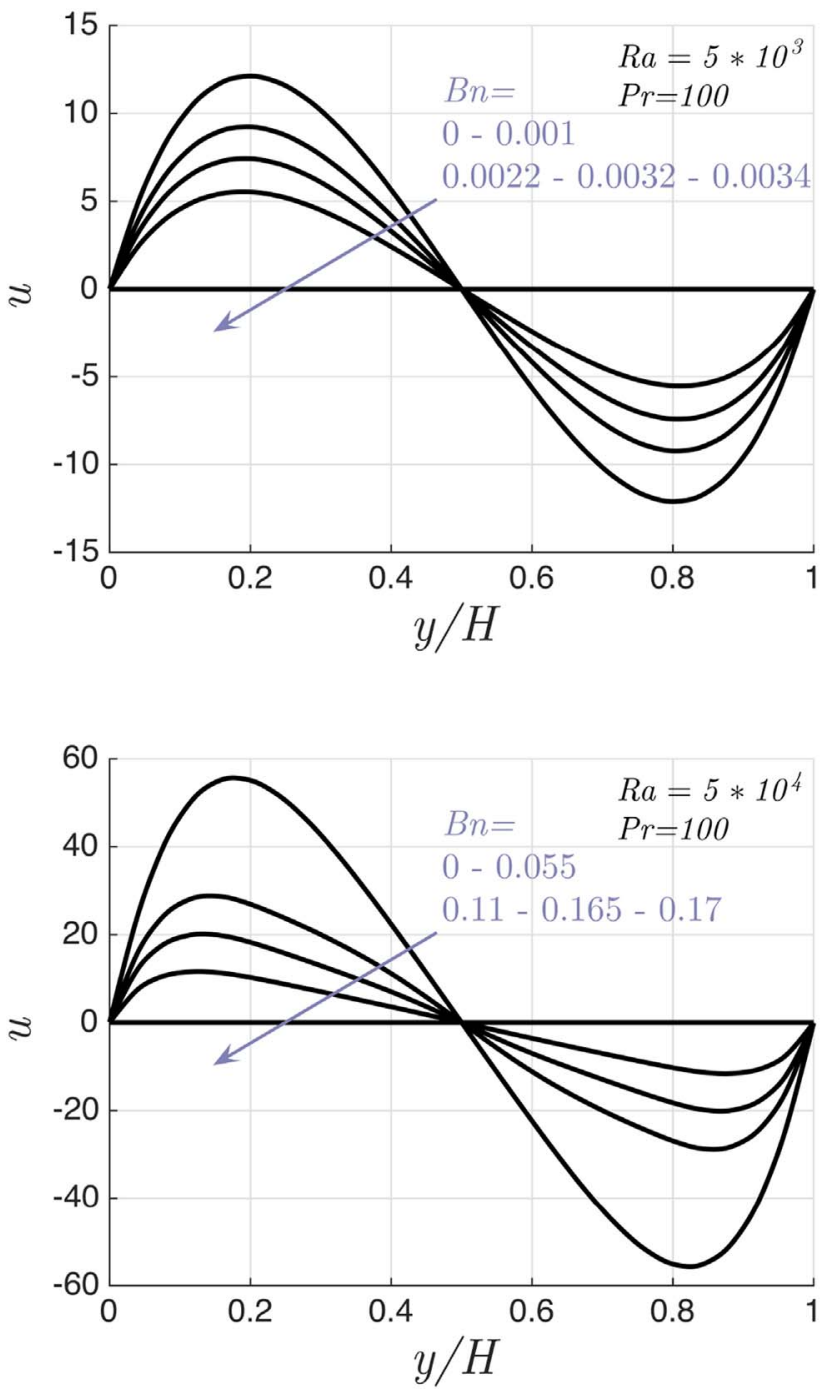

$f_{x}=-\operatorname{Pr}^{\frac{1}{2}} R a^{\frac{-1}{2}}\left(\frac{\partial \tau_{x x}}{\partial x}+\frac{\partial \tau_{y x}}{\partial y}\right)$

$f_{y}=-\operatorname{Pr}^{\frac{1}{2}} R a^{\frac{-1}{2}}\left(\frac{\partial \tau_{y y}}{\partial y}+\frac{\partial \tau_{x y}}{\partial x}\right)$

$[L]=\left[\begin{array}{cccc}l_{1} & 0 & \frac{\partial}{\partial x} & 0 \\ 0 & l_{1} & \frac{\partial}{\partial y} & -1 \\ \frac{\partial}{\partial x} & \frac{\partial}{\partial y} & -\frac{1}{\lambda} & 0 \\ 0 & 0 & 0 & l_{2}\end{array}\right]$

$l_{1}=u \frac{\partial}{\partial x}+v \frac{\partial}{\partial y}$

$l_{2}=u \frac{\partial}{\partial x}+v \frac{\partial}{\partial y}-(\text { Ra. Pr })^{-1 / 2}\left(\frac{\partial^{2}}{\partial x^{2}}+\frac{\partial^{2}}{\partial y^{2}}\right)$

The numerical solutions of system (11) with the corresponding boundary conditions are obtained by developing a numerical code based on the Galerkin weighted residual finite element method with quadrilateral, eight nodes elements. The Galerkin weak statement is obtained by minimizing the residual error over the discretized domain [38].

\subsection{Space discretization}

Using the set of shape function $\left\{N_{k}\right\}_{k=1}^{n}$ the velocity components
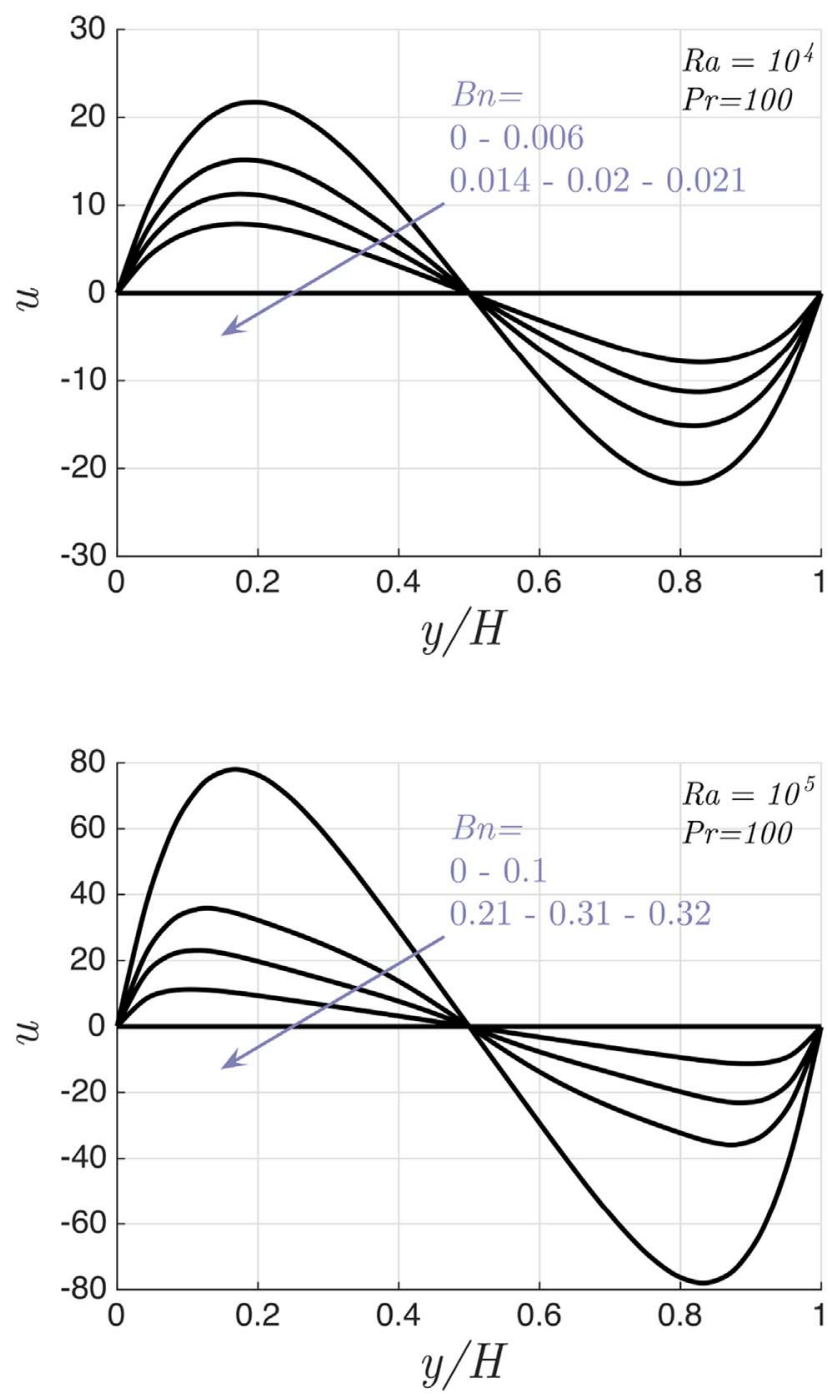

Fig. 6. Variations of non-dimensional velocity $u$ of Casson fluids for different $B n$ values along the vertical mid-plane for different values of $R a$ at $P r=100$. 

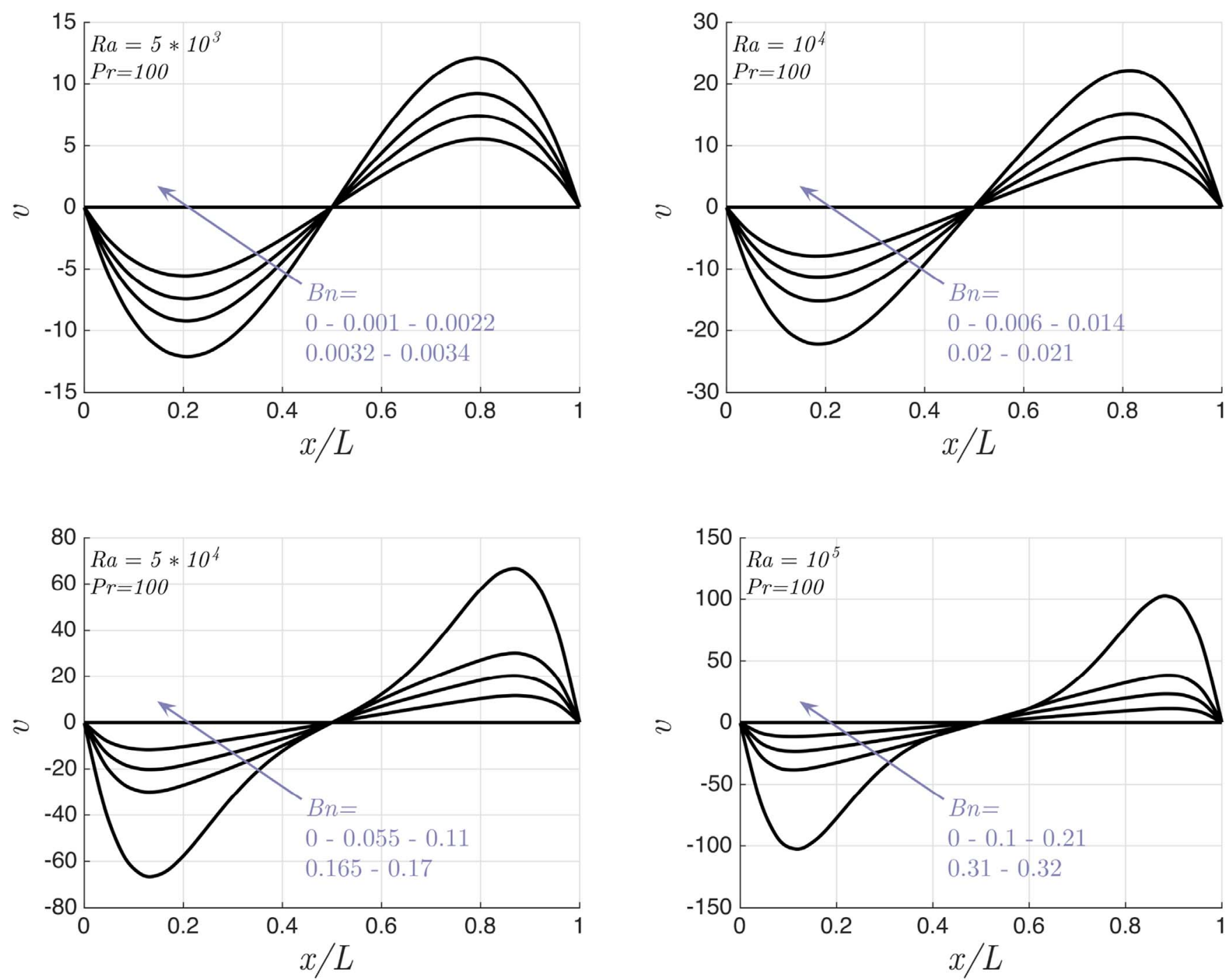

Fig. 7. Variations of non-dimensional velocity $v$ of Casson fluids for different $B n$ values along the horizontal mid-plane for different values of $R a$ at $P r=100$.

$(u, v)$, pressure $p$ and temperature $\theta$ can be expanded in the domain $A(x, y)$ for $(0 \leq x, y \leq 1)$ as bellow:

$u \approx \sum_{k=1}^{n} u_{k} N_{k}(x, y), \quad v \approx \sum_{k=1}^{n} v_{k} N_{k}(x, y)$,

$p \approx \sum_{k=1}^{n} p_{k} N_{k}(x, y), \quad \theta \approx \sum_{k=1}^{n} \theta_{k} N_{k}(x, y)$

where $n$ is the total number of nodes.

Using the quadrilateral, eight nodes elements to discrete the domain and isoparametric method for mapping from $(x, y)$ to a square reference domain $(\xi, \eta)$ one can write:

$x=\sum_{i=1}^{8} x_{i} N_{i}(\xi, \eta), \quad y=\sum_{i=1}^{8} y_{i} N_{i}(\xi, \eta)$

\subsection{Weak formulation}

The integral form of equation (11) can be written as:

$W=\int_{A}\langle\psi\rangle\{[L]\{T\}+\{f\}\} d A=0$

where $\psi$ is a weighting(test) function.

$\langle\psi\rangle=\left\langle\begin{array}{lllll}\psi_{u} & \psi_{v} & \psi_{p} & \psi_{\theta}\end{array}\right\rangle$

Based on the Galerkin weighted residual finite element method the weighting functions $\psi$ comprise all the variations $\delta \mathbf{T}$ of the functions $\mathbf{T}$ : $\psi=\delta \mathbf{T}=\langle N\rangle\{\delta T\}$

Substituting Eq. (19) into Eq. (16) leads to:

$W=\int_{A} \delta \mathbf{T}(\mathcal{L}(\mathbf{T})+f) d A=0$

So

$W=\langle\delta T\rangle \int_{A}\{N\}(\mathscr{L}(\langle N\rangle\{T\})+f) d A=0$

The expansion of Eq. (22) given $W_{i}^{u}, W_{i}^{v}, W_{i}^{p}$ and $W_{i}^{\ominus}$ is detailed in Appendix A. The integrations are evaluate numerically by using a direct integration formula on a square [35] in $(\xi, \eta)$ domain using the following relationships:

$\left\{\begin{array}{l}\frac{\partial N_{i}}{\partial x} \\ \frac{\partial N_{i}}{\partial y}\end{array}\right\}=[J]^{-1}\left\{\begin{array}{l}\frac{\partial N_{i}}{\partial \xi} \\ \frac{\partial N_{i}}{\partial \eta}\end{array}\right\}$

$d x d y=|J| d \xi d \eta$

where $J$ is the Jacobian matrix defined by:

$[J]=\left[\begin{array}{ll}\frac{\partial x}{\partial \xi} & \frac{\partial y}{\partial \xi} \\ \frac{\partial x}{\partial \eta} & \frac{\partial y}{\partial \eta}\end{array}\right]$ 

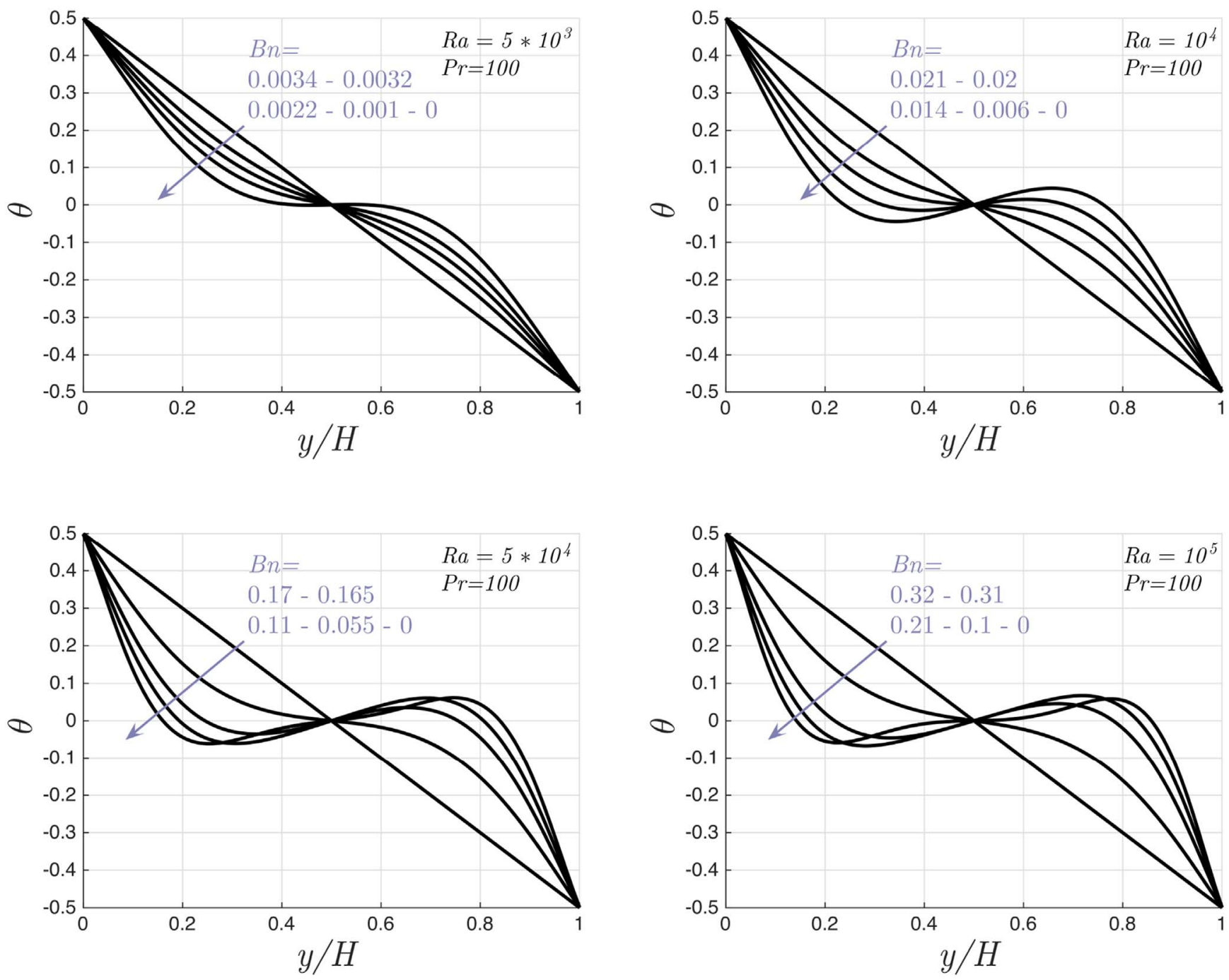

Fig. 8. Variations of non-dimensional Temperature $\theta$ of Casson fluids for different $B n$ values along the vertical mid-plane for different values of $R a$ at $P r=100$.

After applying the numerical integration, a system of algebraic equations can be written based on elementary matrices as follows:

$[K]\{T\}=\{F\}$

Where $[K]$ is the global left hand side matrix, $\{F\}$ is the global load vector and $\{T\}$ is the global unknown vector:

$[K]=\left[\begin{array}{cccc}k_{1}-w k_{2} & 0 & k_{3} & 0 \\ 0 & k_{1}-w k_{2} & k_{4} & -k_{5} \\ k_{3} & k_{4} & -\frac{k_{5}}{\lambda} & 0 \\ 0 & 0 & 0 & k_{1}-(\text { Ra. Pr })^{-1 / 2} k_{2}\end{array}\right]$

where

$$
\begin{aligned}
& k_{1}=u_{p}\{N\} \frac{\partial\langle N\rangle}{\partial x}+v_{p}\{N\} \frac{\partial\langle N\rangle}{\partial y} \\
& k_{2}=-(\{\nabla N\}[H]\langle\nabla N\rangle) \\
& k_{3}=\{N\} \frac{\partial\langle N\rangle}{\partial x} \\
& k_{4}=\{N\} \frac{\partial\langle N\rangle}{\partial y} \\
& k_{5}=\{N\}\langle N\rangle \\
& {[H]=\left[\begin{array}{ll}
1 & 0 \\
0 & 1
\end{array}\right]}
\end{aligned}
$$

$$
\begin{aligned}
\{T\}= & \left\{\begin{array}{c}
u \\
v \\
p \\
\theta
\end{array}\right\} ; \quad\{F\}=-\left\{\begin{array}{c}
f_{n x} \\
f_{n y} \\
0 \\
0
\end{array}\right\} \\
f_{n x}= & -\operatorname{Pr}^{\frac{1}{2}} \operatorname{Ra} \frac{-1}{2}\left(\left[\{N\} \frac{\partial\langle N\rangle}{\partial x}\right]\left\{\tau_{x x}\right\}+\left[\{N\} \frac{\partial\langle N\rangle}{\partial y}\right]\left\{\tau_{y x}\right\}\right) \\
& +w\left(\left[k_{2}\right]\left\{u_{p}\right\}\right) \\
f_{n y}= & -\operatorname{Pr}^{\frac{1}{2}} \operatorname{Ra} \frac{-1}{2}\left(\left[\{N\} \frac{\partial\langle N\rangle}{\partial y}\right]\left\{\tau_{y y}\right\}+\left[\{N\} \frac{\partial\langle N\rangle}{\partial x}\right]\left\{\tau_{x y}\right\}\right) \\
& +w\left(\left[k_{2}\right]\left\{v_{p}\right\}\right)
\end{aligned}
$$

Here $u_{p}$ and $v_{p}$ are the previous values of velocity and $w$ is a constant. At the end of each iteration, the previous values of velocity are replaced by the new computed values: $u_{p}=u$ and $v_{p}=v$. This procedure is used in order to linearize the non-linear terms which come from the advective and convective terms in the momentum and energy equations. The linear system of equation (25) is solved by using the Matlab function 'mldivide'. Using the new computed values, the stress terms are evaluated and the process is repeated until the convergence is reached. The convergence is obtained when the norm 2 of the relative difference between the new and the previous values of the vector $\{T\}$ is less than $10^{-5}$.

The velocity distribution of Newtonian fluids is considered as the initial values of $u_{p}$ and $v_{p}$. 


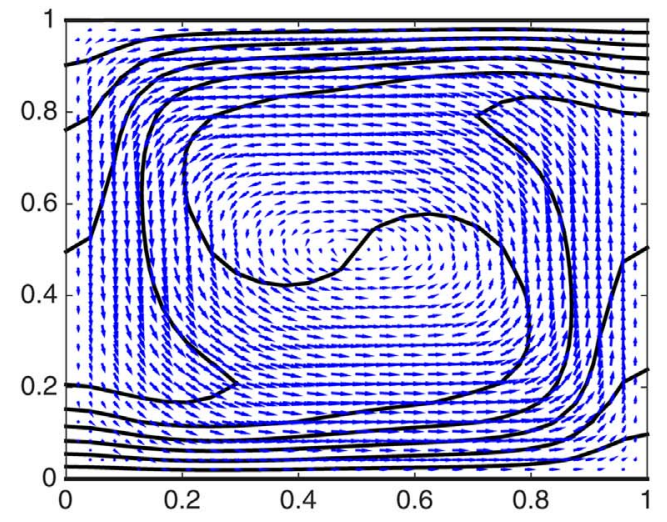

(a) $B n=0$

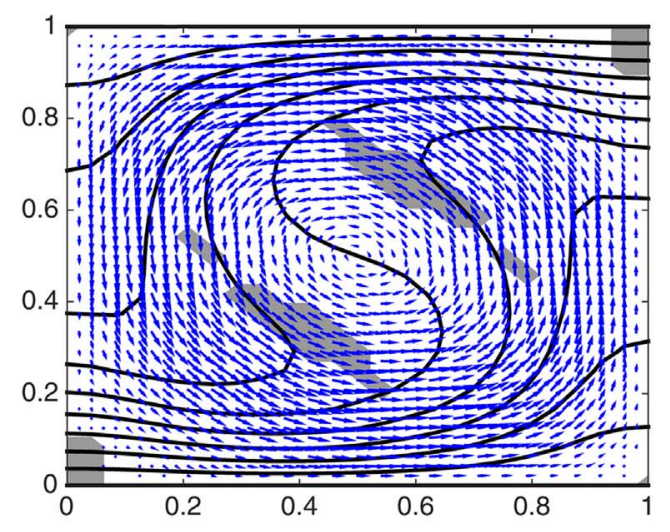

(c) $B n=0.12$

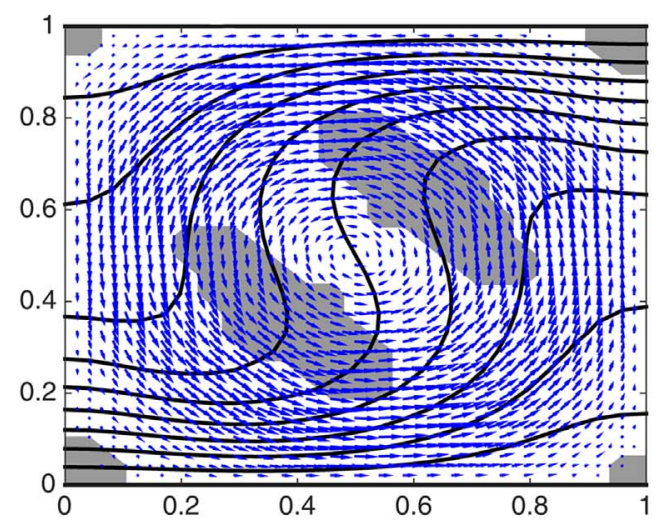

(e) $B n=0.24$

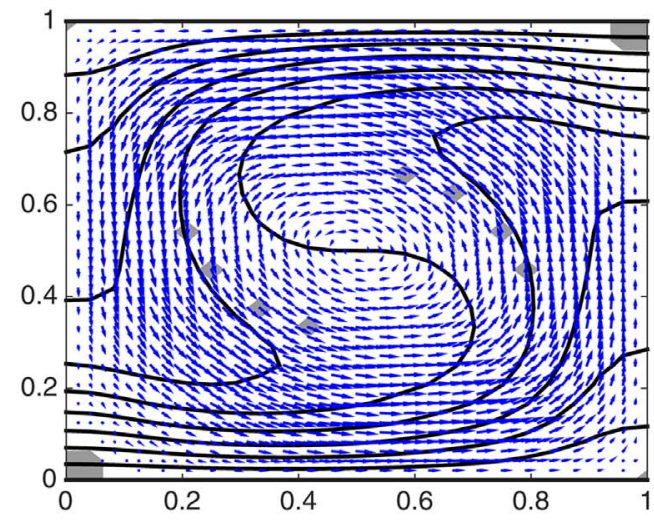

(b) $B n=0.06$

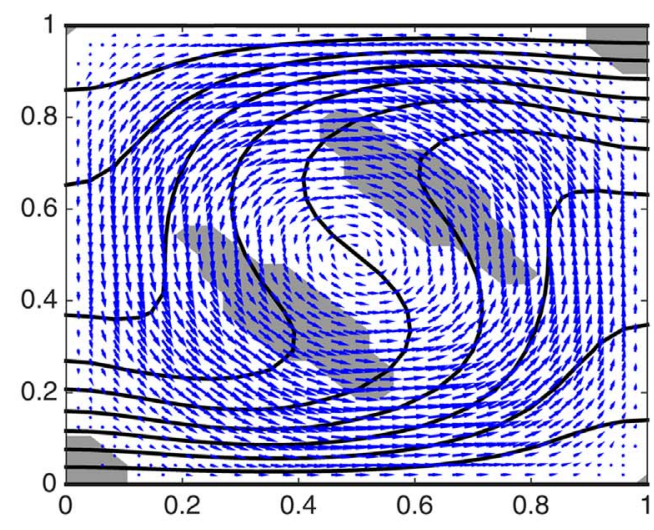

(d) $B n=0.18$

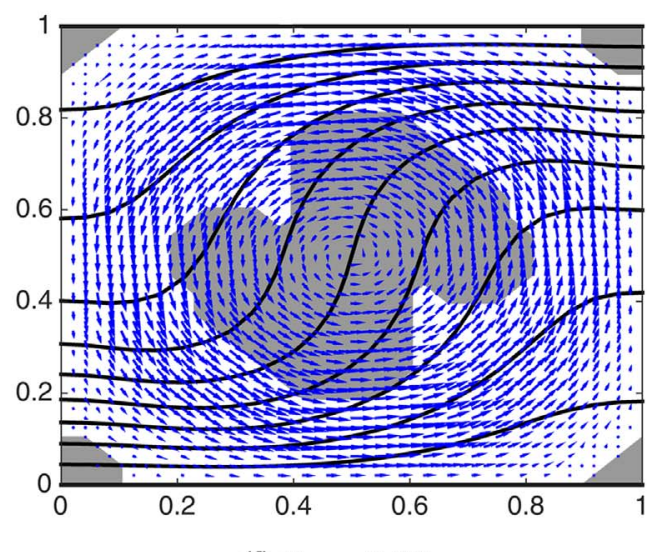

(f) $B n=0.30$

Fig. 9. Contours of non-dimensional temperature $\theta$ and velocity vectors with plug regions of Casson fluids for six different values of Bingham number at $R a=10^{5}$ and $P r=100$.

It is worth mentioning that the numerical analysis of Eq. (10) leads to an unstable system of equations. In order to circumvent this difficulty, the Laplacian velocity components were added in Eqs. (26) and (29). Because these terms are added on both sides of the momentum equations, they vanish by considering the whole system. It means that these terms do not affect the results but allow to make the equations stable to introduce diffusive terms which make the equation stable.

\subsection{Numerical method validation}

The grid independent solution has been tested by considering two cases $\left(R a=10^{5}, \operatorname{Pr}=10, B n=0\right)$ and $\left(R a=10^{5}, \operatorname{Pr}=1000, B n=0\right)$. The convergence is tested on the values of the average Nusselt number. We found that the mesh consisting in 1825 nodes guarantees a grid independent solution within the relative tolerance level of $10^{-5}$.

In order to ensure convergence of results in the case of viscoplastic fluid, the convergence of the solutions was checked by varying the penalty and regularization parameters. Results show that $\overline{\mathrm{Nu}}$ converges within $0.1 \%$ by varying $\lambda$ from $10^{3}$ to $10^{4}$ and also by varying $m$ from $10^{3}$ to $10^{4}$. In the following, all results are obtained for $\lambda=10^{4}$ and $m=10^{4}$.

The numerical method has been validated by considering the Newtonian case $(B n=0)$. Our results have been compared with that ones given in Refs. [37], [39]. A very good agreement has been obtained within a mean relative difference of $1 \%$.

The numerical code was also validated by testing the Bingham case. Results of mean Nusselt number $\overline{N u}$ for $R a=5 * 10^{4}$ and $P r=10$ are shown in Fig. 3, one observes a good agreement between our results (continuous line) and the results obtained by Turan et al. [23] (circles). 

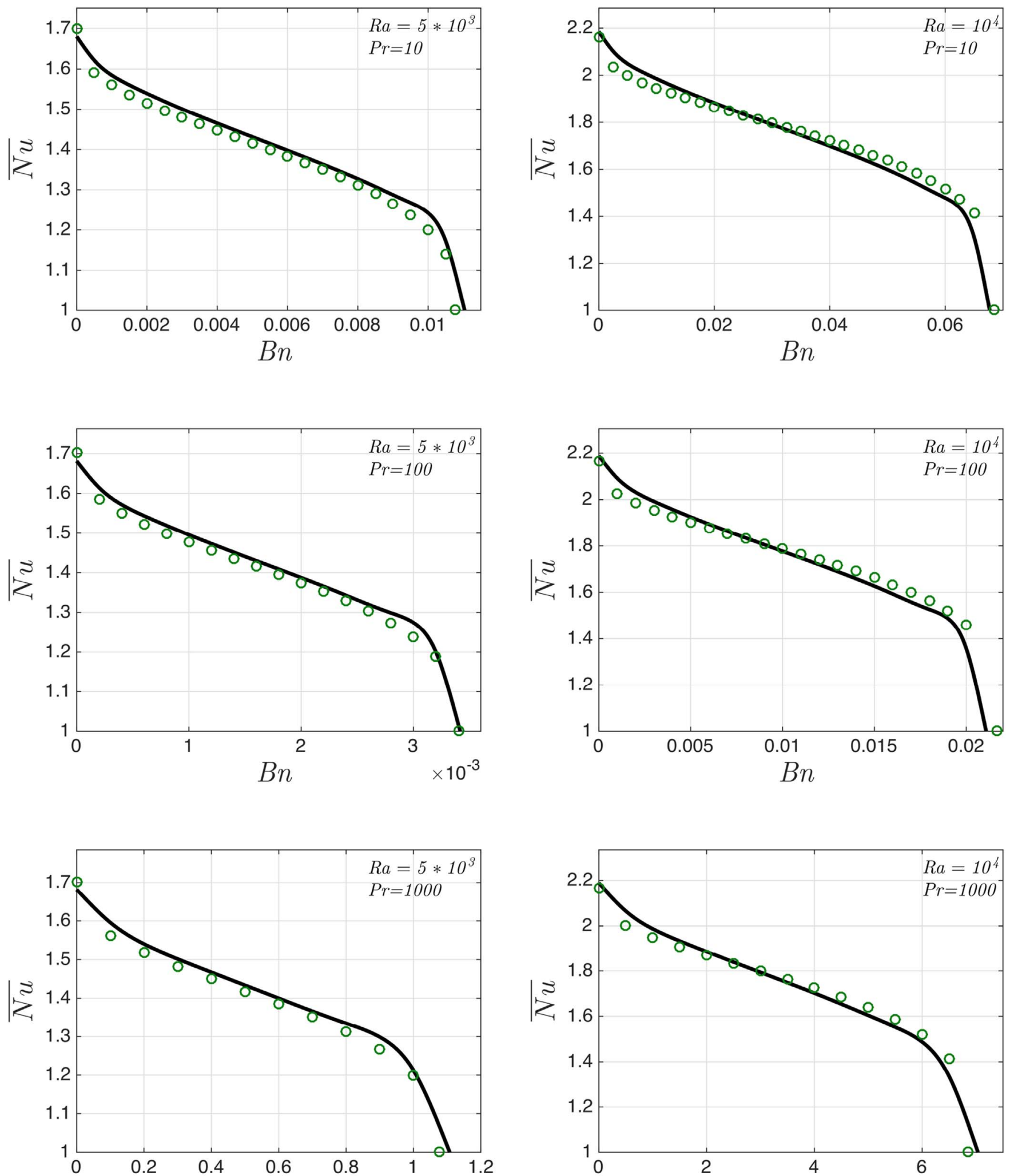

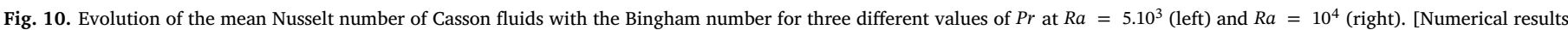
$(-)$ and prediction of the correlation (o)given by Eq. (30)].

\section{Results and discussion}

In this part, we only consider the Rayleigh-Bénard Convection of Casson fluids. One knows that the RBC occurs when buoyancy effects are larger than the viscous and thermal diffusivity effects (dissipative effects). It means that above a critical $R a$ value, convection occurs. In the case of Casson fluids, the onset of convection is depicted in Fig. 4. This figure displays the domain in the $\left(R a_{c}, B n\right)$ parametric plane where convection cells are possible for different values of $\mathrm{Pr}$, i.e. when $R a \geq R a_{c}$. One notices that the critical Rayleigh number increases with 

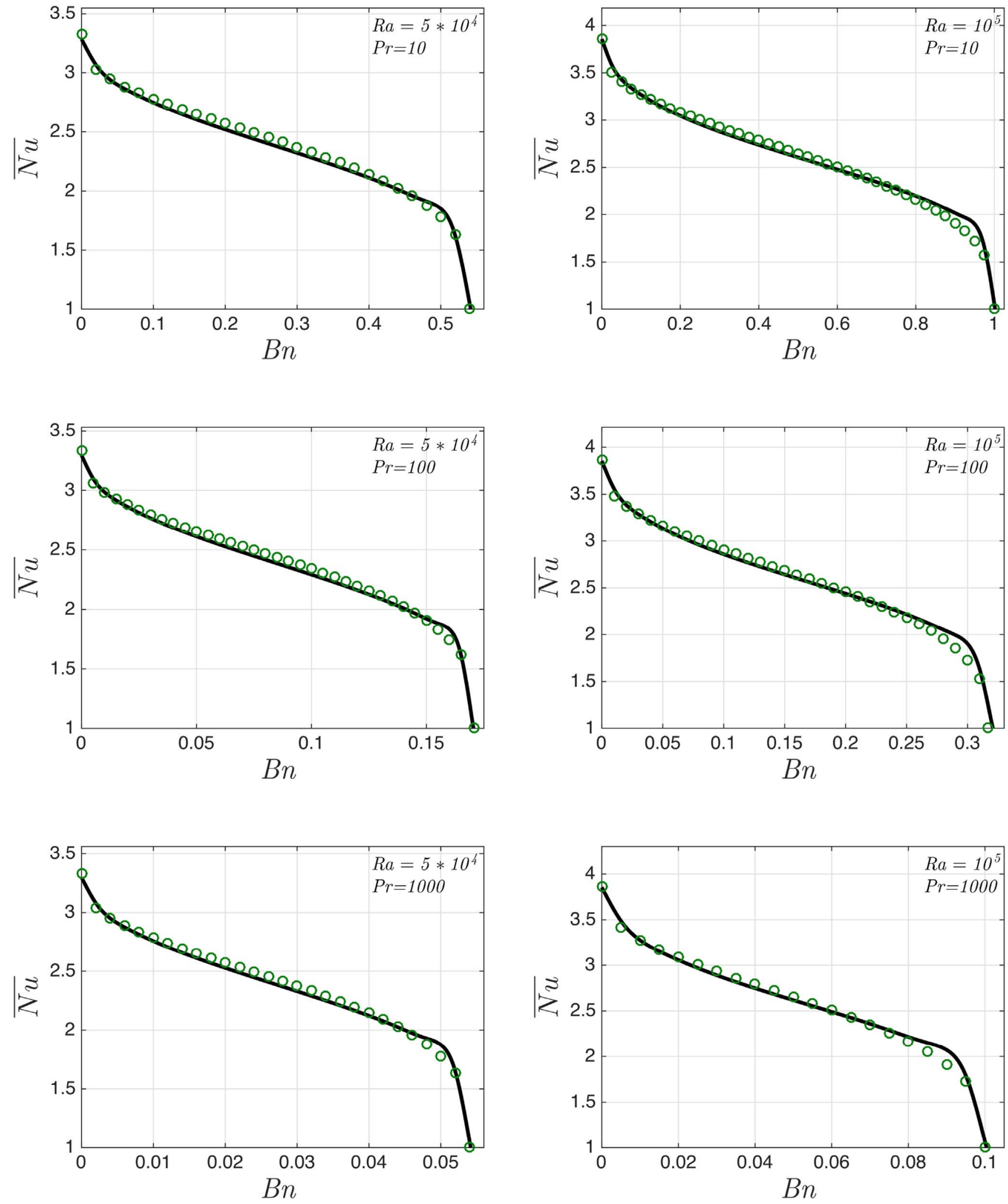

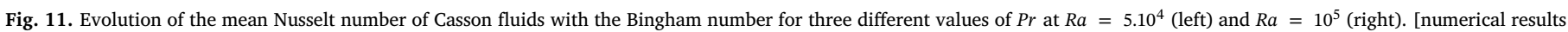
$(-)$ and prediction of the correlation (o)given by Eq. (30)].

$B n$ at fixed $P r$. It highlights a stabilizing effect of the yield stress via $B n$. Similarly, the increase in $\mathrm{Pr}$ stabilizes the motionless solution. In order to compare our results to experimental studies given by Refs. [29] [30], and [32], Fig. 5 represents the onset of convection in terms of the critical Yield number $Y_{c}$ (where the Yield number is defined as $Y=\frac{\tau_{y}}{\rho \beta g \Delta T H}$
) as a function of the Rayleigh number for $P r=100$. In the range of the Rayleigh values involved in the experimental studies $\left(R a=O\left(10^{4}\right)-O\left(10^{5}\right)\right)$, we obtain realistic values of $Y_{c}$ since in Refs. [29] and [32], the studies highlight $1 / Y_{c} \approx 80$ (i.e. $Y_{c} \approx 10^{-2}$ ) for no-slip conditions and (perfect) conductive horizontal walls. Furthermore, it is 
worth noting that criticality is sensitive to boundary conditions and the value of aspect ratio. In Refs. [29] and [32], several values of aspect ratio are investigated between 6 and 18, while in the present study, the aspect ratio has been chosen equal to 1 corresponding to the benchmark case of the Rayleigh-Bénard configuration. When considering slip boundary conditions, the value of $1 / Y_{c}$ decreases as highlighted by Ref. [29]. In Ref. [30], results provide that $1 / Y_{c} \approx 5$ for slip conditions and insulated horizontal walls conditions which are quite far from the present study.

\subsection{Velocity and temperature}

When the convection occurs, the velocity vectors are no longer equal to zero as well as the linear profile of the temperature is modified by the motion. Figs. 6 and 7 depict the variations of non-dimensional horizontal component of velocity $u$ and non-dimensional vertical component of velocity $v$ along the vertical and horizontal mid-planes of the cavity when $P r=100$ and for different values of $R a$ and $B n$. Results show that the magnitude of velocity increase with increasing $R a$ due to stronger buoyancy force. In other words, the increase in $R a$ strengthens the intensity of convection. On the other hand, one can see that the magnitudes of velocity and temperature, displayed in Figs. 6-8, decrease with increasing Bingham number indicating the stabilizing effect of the yield stress, which corresponds to an additional flow resistance. This result agrees with those obtained in the Bingham and HerschelBulkley cases [23] [26], [27]. In these articles, it is shown that the increase in the Bingham number (or Yield number) leads to stop the convective motion. It means that for fixed $R a$ and $P r$ values, there exists a value of $B n$ above which the convection does not occur, say $B n_{\max }$. Figs. 6-8 show that the value of $B n_{\max }$ increases with increasing $R a$ due to the stronger convection force which can overcome the flow resistance up to greater values of the Bingham number.

Similarly, variations of non-dimensional temperature $\theta$ with $R a$ and $B n$ along the vertical mid-plane of the cavity have been shown in Fig. 8. As expected, the non-linearity of temperature distribution increases with increasing $R a$ due to stronger convection force. On the other hand, the variation of the temperature $\theta$ tend to a linear profile when the value of Bingham number increases and reaches $B n_{\max }$, meaning that the heat transfer is only due to thermal conduction and there is no flow within the enclosure.

\subsection{Contours of temperature and velocity vectors}

Fig. 9 shows contours of non-dimensional temperature $\theta$ and velocity vectors for different values of Bingham number at $R a=10^{5}$ and $P r=100$. The Shaded areas in these figures show the unyielded regions where $\tau<\tau_{y}$. One can observe different types of regions: the Apparently Unyielded Regions (AUR) and the Truly Unyielded Regions (TUR) [35]. At the corners of the cavity, the unyielded regions correspond to AUR since they are dead zones where the velocities are very small and the fluid is stagnant. In the center of the cavity which coincides with the center of the convective cell, TUR appear when $B n$ is increased at nominal $R a$ and $P r$ values as one can see in Fig. 9. The unyielded regions occur where the shear rate is minimal (equal to zero). By contrast, maximal values of shear rate are located at walls where no slip conditions are considered. The increase in $B n$ is accompanied by an increase in the TUR area as well as a decrease in the convective intensity. Finally, unyielded regions invade the whole cavity at $B n=B n_{\max }$. Obviously, the increase in TUR area highlights the decrease in the convection intensity as already discussed in the paragraph 4.1 . These results are in agreement with results proposed by Turan et al. [23] for Bingham fluids and by Refs. [26] [27],for Herschel-Bulkley fluids.

\subsection{Heat transfer (Nusselt number)}

The variations of mean Nusselt number $\overline{N u}$ with Bingham number
$B n$ are shown in Figs. 10 and 11 for different values of Prandtl number $(P r=10,100,1000)$ at respectively $R a=5.10^{3}, 10^{4}$ and $R a=5.10^{4}, 10^{5}$. It can be seen that the maximal value of the mean Nusselt number occurs for Newtonian fluids where $B n=0$. For viscoplastic fluids $(B n>0)$, the mean Nusselt number decreases with increasing Bingham number, underlying once again that the yield stress decreases the convective motion intensity. As already discussed, for a given $\mathrm{Ra}$ and $\mathrm{Pr}$ set of values, results show that the mean Nusselt number decreases with increasing Bingham number and for large value of Bingham number $B n \geq B n_{\max }$ the value of mean Nusselt number fall abruptly to $\overline{N u}=1$, meaning that convection stops leading to a motionless state. The transition between the convective to conductive regimes is abrupt in terms of Nusselt values, highlighting a subcritical bifurcation which is in agreement with [31] and [27]. As expected, at fixed value of $B n$, the increase in the Rayleigh number involves larger heat transfer ( $\overline{\mathrm{Nu}}$ values) via larger buoyancy effects. It also means that $B n_{\max }$ increases with increasing $R a$.

Concerning the influence of the $\mathrm{Pr}$ number, one notices that results are insensitive to $\operatorname{Pr}$ variations in the Newtonian case. However, for viscoplastic fluids $(B n \neq 0)$, the increase in $\operatorname{Pr}$ values decreases the heat transfer as well as the value of $B n_{\max }$ as displayed in Figs. 10 and 11.

Our results obtained for Casson fluids can be compared with the results obtained for Bingham [23] and the shear-thinning HerschelBulkley fluids [17]. At fixed $R a$ and $B n$ values, $P r=10$, one can observe that Casson fluids lead to smaller $\overline{\mathrm{Nu}}$ values than the two other models which involves a larger stabilizing effect of the yield stress in the case of Casson fluids. In other words, the heat transfer is the smallest one for the Casson fluids while the largest heat transfer is obtained for Herschel-Bulkley model. This result is correlated with the non-linear (shear-thinning) variations of the viscosity [37], [40].

\subsection{Correlation for the mean Nusselt number}

A correlation for the mean Nusselt number $\overline{N u}$ can be proposed in a general form as below:

$\overline{N u}=f(R a, \operatorname{Pr}, B n)=1+\left(\overline{N u}_{0}-1\right)\left[1-\left(\frac{B n}{B n_{\max }}\right)^{0.3}\right]^{a}$

where $\overline{N u}_{0}$ is the mean Nusselt number obtained in the Newtonian case $(B n=0)$ and $B n_{\max }$ is the Bingham number above which the convection does not occur and $\overline{\mathrm{Nu}}=1$. The values of $\overline{\mathrm{Nu}}_{0}$ and $B n_{\max }$ can be estimated by fitting our numerical results:

$\overline{N u}_{0}=0.3495 *(\ln (R a)-5.125)^{1.296} *\left[\frac{P r}{P r+1}\right]^{0.02}$
$B n_{\text {max }}=(0.03984 * \ln (R a)-0.3207)^{1.8} * R a^{0.3097} *\left[\frac{P r}{10}\right]^{-0.5}$

and

$a=-0.01537 *(\ln (R a)-15.73)^{3}-0.2263 *(\ln (R a)-13.42)^{2}$

The correlation we propose for $\overline{N u}_{0}$ in Eq. (31) implies a maximal relative difference with our numerical results which is less than $2 \%$ and a mean relative difference smaller than $1 \%$ for $5.10^{3}<R a<10^{5}$ and $\operatorname{Pr}=10,100,1000$ as one can observe in Figs. 10 and 11. These correlations are also very useful in the case of viscoplastic fluids, since they provide the heat transfer via Eq. (30) and also $B n_{\max }$ (Eq. (32)), the value above which the flow cannot occur. This last value is determined as a function of $R a$ and $P r$, with a maximal relative difference with our numerical results of $3.2 \%$ and a mean relative difference close to $1 \%$.

\section{Conclusions}

In this study, a finite element numerical code has been developed in order to investigate the Rayleigh-Bénard convection in viscoplastic 
fluids modeled by a Casson law. A two-dimensional square enclosure is considered. Results obtained for a Casson fluid are qualitatively similar to that obtained for Bingham and Herschel-Bulkley fluids. It means that the stabilizing effect of the yield stress via the Bingham number is highlighting since the increase in $B n$ reduces the convection intensity. From a threshold value, $B n_{\max }$, the convective motion stops leading to a conductive state characterized by a linear temperature profile and $\overline{\mathrm{Nu}}=1$. One recovers that the transition between the conductive to the convective state $\left(B n<B n_{\max }\right.$ ) is abrupt corresponding to a subcritical bifurcation. Furthermore, we also obtain that the truly unyielded regions appear in the center of the convective cell where the shear rate is minimal. By contrast, maximal values of shear rate are reached at walls. The areas of unyielded regions increase with the increase in $B n$, unyielded regions invade the whole cavity from $B n_{\max }$. Finally, the heat transfer is evaluated and correlations for the mean Nusselt number as well as $B n_{\max }$ are provided as a function of $R a, P r$ and $B n$. By comparing with the Bingham and Herschell-Bulkley models, the Casson model leads to the smallest heat transfer.

\section{Appendix A. Finite element analysis - Weak formulation}

$$
\begin{aligned}
W_{i}^{u}=\sum_{k=1}^{n} u_{k} \int_{A}\left[\left(\sum_{k=1}^{n} u_{k} N_{k}\right) \frac{\partial N_{k}}{\partial x}+\left(\sum_{k=1}^{n} v_{k} N_{k}\right) \frac{\partial N_{k}}{\partial y}\right] N_{i} d x d y \\
+\sum_{k=1}^{n} p_{k} \int_{A}\left(\frac{\partial N_{k}}{\partial x}\right) N_{i} d x d y \\
\quad-\operatorname{Pr} \frac{1}{2} R a^{\frac{-1}{2}} \int_{A}\left[\left(\sum_{k=1}^{n} \tau_{x x_{k}} \frac{\partial N_{k}}{\partial x}\right)+\left(\sum_{k=1}^{n} \tau_{y x_{k}} \frac{\partial N_{k}}{\partial y}\right)\right] N_{i} d x d y \\
W_{i}^{\nu}=\sum_{k=1}^{n} v_{k} \int_{A}\left[\left(\sum_{k=1}^{n} u_{k} N_{k}\right) \frac{\partial N_{k}}{\partial x}+\left(\sum_{k=1}^{n} v_{k} N_{k}\right) \frac{\partial N_{k}}{\partial y}\right] N_{i} d x d y \\
\quad+\sum_{k=1}^{n} p_{k} \int_{A}\left(\frac{\partial N_{k}}{\partial y}\right) N_{i} d x d y-\sum_{k=1}^{n} \theta_{k} \int_{A}\left(N_{k}\right) N_{i} d x d y \\
\quad-\operatorname{Pr}^{\frac{1}{2}} \operatorname{Ra} \frac{-1}{2} \int_{A}\left[\left(\sum_{k=1}^{n} \tau_{y y_{k}} \frac{\partial N_{k}}{\partial y}\right)+\left(\sum_{k=1}^{n} \tau_{x y_{k}} \frac{\partial N_{k}}{\partial x}\right)\right] N_{i} d x d y
\end{aligned}
$$

$$
\begin{gathered}
W_{i}^{p}=\sum_{k=1}^{n} u_{k} \int_{A}\left(\frac{\partial N_{k}}{\partial x}\right) N_{i} d x d y+\sum_{k=1}^{n} v_{k} \int_{A}\left(\frac{\partial N_{k}}{\partial y}\right) N_{i} d x d y \\
-\frac{1}{\lambda} \sum_{k=1}^{n} p_{k} \int_{A}\left(N_{k}\right) N_{i} d x d y
\end{gathered}
$$

And

$$
\begin{gathered}
W_{i}^{\theta}=\sum_{k=1}^{n} \theta_{k} \int_{A}\left[\left(\sum_{k=1}^{n} u_{k} N_{k}\right) \frac{\partial N_{k}}{\partial x}+\left(\sum_{k=1}^{n} v_{k} N_{k}\right) \frac{\partial N_{k}}{\partial y}\right] N_{i} d x d y \\
+(\text { Ra. Pr })^{-1 / 2} \sum_{k=1}^{n} \theta_{k} \int_{A}\left[\frac{\partial N_{i}}{\partial x} \frac{\partial N_{k}}{\partial x}+\frac{\partial N_{i}}{\partial y} \frac{\partial N_{k}}{\partial y}\right] d x d y
\end{gathered}
$$

\section{References}

[1] E. Bingham, The behavior of plastic materials, Bull US Bur Stand 13 (1916) 309-353.

[2] W.H. Herschel, R. Bulkley, Measurement of consistency as applied to rubber-benzene solutions, Am Soc Test Proc 26 (2) (1926).

[3] N. Casson, Rheology of disperse systems, Pergamon Press, Oxford, 1959.

[4] J.F. Steffe, Rheological methods in food process engineering, Freeman Press, 1996.

[5] G.W. Scott Blair, The success of Casson's equation, Rheol Acta 5 (3) (Sep. 1966) 184-187.

[6] N. Iida, Influence of plasma layer on steady blood flow in microvessels, Jpn J Appl Phys 17 (1) (Jan. 1978) 203-214.

[7] P. Chaturani, R.P. Samy, Pulsatile flow of Casson's fluid through stenosed arteries with applications to blood flow, Biorheology 23 (5) (1986) 499-511.

[8] R.K. Dash, G. Jayaraman, K.N. Mehta, Estimation of increased flow resistance in a narrow catheterized artery-a theoretical model, J Biomech 29 (7) (Jul. 1996) 917-930.

[9] S. Chakravarty, Sarifuddin, P.K. Mandal, Unsteady flow of a two-layer blood stream past a tapered flexible artery under stenotic conditions, Comput Methods Appl Math 4 (4) (2004) 391-409.

[10] D.S. Sankar, U. Lee, Two-fluid Casson model for pulsatile blood flow through stenosed arteries: a theoretical model, Commun Nonlinear Sci Numer Simul 15 (8) (2010) 2086-2097.

[11] C.W. Macosko, Rheology : principles, measurements, and applications, VCH, 1994.

[12] R.P. Chhabra, J.F. Richardson, Non-Newtonian flow and applied rheology, Elsevier, 2008.

[13] S. Chandrasekhar, Hydrodynamic and hydromagnetic stability, Clarendon, Oxford, 1961.

[14] E.L. Koschmieder, Bénard cells and taylor vortices, Cambridge University Press, 1993.

[15] E. Bodenschatz, W. Pesch, G. Ahlers, Recent developments in Rayleigh-bénard convection, Annu Rev Fluid Mech 32 (1) (Jan. 2000) 709-778.

[16] H.M. Park, Rayleigh-Bénard convection of nanofluids based on the pseudo-singlephase continuum mode, Int J Therm Sci 90 (2015) 267-278.

[17] M.S. Aghighi, A. Ammar, C. Metivier, F. Chinesta, Parametric solution of the Rayleigh-Benard convection model by using the PGD Application to nanofluids, Int J Numer Methods Heat Fluid Flow 25 (6) (2015) 1252-1281.

[18] C. Sanmiguel Vila, S. Discetti, G.M. Carlomagno, T. Astarita, A. Ianiro, On the onset of horizontal convection, Int J Therm Sci 110 (2016) 96-108.

[19] N. Yada, P. Kundu, S. Paul, P. Pal, Different routes to chaos in low Prandtl-number Rayleigh-Bénard convection, Int J Non Linear Mech 81 (2016) 261-267.

[20] P. Kaur, J. Singh, Heat transfer in thermally modulated two-dimensional Rayleigh Bénard convection, Int J Therm Sci 114 (2017) 35-43.

[21] J. Zhang, D. Vola, I. a. Frigaard, Yield stress effects on Rayleigh-Bénard convection, J Fluid Mech 566 (Oct. 2006) 389-419.

[22] a. Vikhansky, Thermal convection of a viscoplastic liquid with high Rayleigh and Bingham numbers, Phys Fluids 21 (10) (2009) 103103.

[23] O. Turan, N. Chakraborty, R.J. Poole, Laminar Rayleigh-Bénard convection of yield stress fluids in a square enclosure, J Nonnewton Fluid Mech. 171-172 (Mar. 2012) 83-96.

[24] S. Yigit, R.J. Poole, N. Chakraborty, Effects of aspect ratio on natural convection of Bingham fluids in rectangular enclosures with differentially heated horizontal walls heated from below, Int J Heat Mass Transf 80 (Jan. 2015) 727-736.

[25] M.A. Hassan, M. Pathak, M.K. Khan, Rayleigh-Benard convection in HerschelBulkley fluid, J Nonnewton Fluid Mech. 226 (2015) 32-45.

[26] M.S. Aghighi, A. Ammar, Aspect ratio effects in Rayleigh-Bénard convection of Herschel-Bulkley fluids, Eng Comput 34 (5) (Jul. 2017) 1658-1676.

[27] C. Li, A. Magnin, C. Métivier, Natural convection in shear-thinning yield stress fluids in a square enclosure, AIChE J 62 (4) (Apr. 2016) 1347-1355.

[28] S. Yigit, S. Chen, P. Quinn, N. Chakraborty, Numerical investigation of laminar Rayleigh-Bénard convection of Bingham fluids in square cross-sectioned cylindrical enclosures, Int J Therm Sci 110 (2016) 356-368.

[29] M. Darbouli, C. Métivier, J.-M. Piau, A. Magnin, A. Abdelali, Rayleigh-Bénard convection for viscoplastic fluids, Phys Fluids 25 (2) (2013) 23101.

[30] Z. Kebiche, C. Castelain, T. Burghelea, Experimental investigation of the RayleighBénard convection in a yield stress fluid, J Nonnewton Fluid Mech. 203 (Jan. 2014) 9-23.

[31] A. Vikhansky, On the stopping of thermal convection in viscoplastic liquid, Rheol Acta 50 (4) (Apr. 2011) 423-428. 
[32] C. Métivier, C. Li, A. Magnin, Origin of the onset of Rayleigh-Bénard convection in a concentrated suspension of microgels with a yield stress behavior, Phys Fluids 29 (10) (2017).

[33] A. Davaille, B. Gueslin, A. Massmeyer, E. Di Giuseppe, Thermal instabilities in a yield stress fluid: existence and morphology, J Nonnewton Fluid Mech 193 (2013) 144-153.

[34] T.C. Papanastasiou, Flows of materials with yield, J Rheol (N Y N Y) 31 (5) (Jul. 1987) 385.

[35] E. Mitsoulis, Flows of viscoplastic materials: models and computations, Rhetor Rev 2007 (2007) 135-178.

[36] W. Ibrahim, O.D. Makinde, Magnetohydrodynamic stagnation point flow and heat transfer of casson nanofluid past a stretching sheet with slip and convective boundary condition, J Aerosp Eng (2015) 1-11.

[37] M.S. Aghighi, A. Ammar, C. Metivier, M. Normandin, F. Chinesta, Non-incremental transient solution of the Rayleigh-Bénard convection model by using the PGD, J Nonnewton Fluid Mech 200 (Oct. 2013) 65-78.

[38] G. Dhatt, G. Touzot, E. Lefrancois, Finite element method, Wiley, 2012.

[39] N. Ouertatani, N. Ben Cheikh, B. Ben Beya, T. Lili, Numerical simulation of twodimensional Rayleigh-Bénard convection in an enclosure, Compt Rendus Mec 336 (5) (May 2008) 464-470.

[40] O. Turan, A. Sachdeva, N. Chakraborty, R.J. Poole, Laminar natural convection of power-law fluids in a square enclosure with differentially heated side walls subjected to constant temperatures, J Nonnewton Fluid Mech. 166 (17-18) (Sep. 2011) 1049-1063. 